\title{
Posterior malleolar fractures. New classification and treatment algorithm
}

\author{
Diego Yearson ${ }^{1,23}\left(\mathbb{D}\right.$, Ignacio Melendez ${ }^{1,2}\left(\mathbb{D}\right.$, Federico Anain ${ }^{(\mathbb{D})}$, Santiago Siniscalchi' ${ }^{(\mathbb{D})}$, Juan Drago ${ }^{1,2,3}$ (i) \\ 1. Sanatorio de la Trinidad Ramos Mejía, Buenos Aires, Argentina. \\ 2. Sanatorio de la Trinidad Palermo, Buenos Aires, Argentina. \\ 3. CLIMBA Centro de Traumatología, Buenos Aires, Argentina.
}

\section{Abstract}

Objective: This study proposes a new classification of posterolateral malleolar fractures and a treatment algorithm.

Methods: We divided the posterolateral malleolus, which we considered as the posterior malleolus, from the posteromedial one, which we considered as being part of the medial malleolus fracture. The experience with 77 patients treated from February 2017 to February 2020 was assessed. All of them were assessed by frontal and profile radiographies and computed tomography (CT). Among the parameters to classify these fractures, we believe the most determining ones are fracture size, followed by presence of fracture displacement.

Results: Fractures were divided into those whose posterior fragment was $25 \%$ smaller than the tibial joint surface and those that compromised more than $25 \%$ of this joint. The first group underwent syndesmotic opening and was subclassified into $1 \mathrm{~A}$ (stable fractures), which do not require surgical treatment, and 1B (unstable), which require syndesmotic stabilization. The second group, which comprised the larger fractures, was subclassified into 2 A (non-displaced fractures, or with a displacement below $2 \mathrm{~mm}$ ), which underwent percutaneous osteosynthesis, 2B (displaced fractures), and 2C (comminuted fractures), which underwent open reduction and internal fixation using a posterior approach.

Conclusion: The classifications published so far are anatomic or descriptive, but none of them proposes a therapeutic algorithm for each type of fracture. We believe it will be helpful for its interpretation and decision-making on the need to perform a posterior approach, prioritizing the anatomical reduction of the joint fragment and resolution of syndesmotic instability linked to each fracture pattern using the most simple and effective method.

Level of Evidence IV; Therapeutic Studies; Case Series.

Keywords: Ankle fractures/classification; Algorithms; Treatment outcomes.

\section{Introduction}

The treatment of posterior malleolar fractures of the ankle has been changing throughout time, especially in the last 10 years. According to worldwide statistics, posterior malleolar fractures are associated with ankle fractures in 7 to $44 \%$ of the cases and usually worsen the prognosis of these fractures ${ }^{(1)}$.

The scarce relevance historically given to the association between these two injuries made that concerns to identify a specific pattern for malleolar fractures were raised only a few years ago, to classify and improve their post-treatment clinical outcomes.

Initially, these fractures were underestimated and did not receive surgical treatment ${ }^{(2)}$. Later, patients began to undergo anterior compression screw osteosynthesis( ${ }^{(3)}$ with indirect reduction without approaching the fracture focus; finally, in the last decade, the prevailing idea has been to approach the fracture posteriorly and thus to perform open reduction and internal fixation. Good initial outcomes lead to the indication of posterior reduction to practically all posterior malleoli, with no actual parameters to standardize this intervention. Placing patients in the prone position and adding a new approach should be justified, to provide the patient with an actual benefit and not bringing an additional element of morbidity.

The main research question is to investigate whether a posterior approach is required in all cases ${ }^{(4)}$, which implies in pre-operative planning.
Study performed at the Trinidad Ramos Mejia, Ramos Mejia, Buenos Aires, Argentina.

Correspondence: Diego Javier Yearson. 2021 Maure St., Buenos Aires, Argentina, Zip Code: 1426. E-mail: dyearson@intramed.net. Conflicts of interest: none. Source of funding: none. Date received: September 16, 2020. Date accepted: November 09, 2020. Online: December 21, 2020

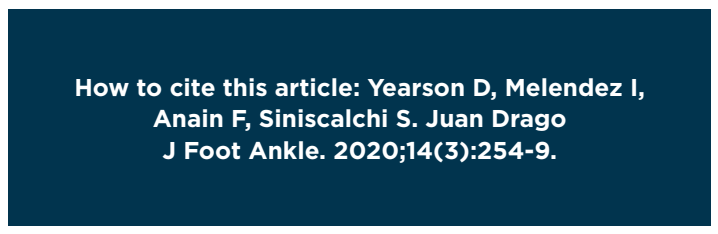




\section{Methods}

This study was approved by the Institutional Review Board, and a level IV retrospective study was conducted.

All patients with $\mathrm{AO} 44 \mathrm{~B}$ and $44 \mathrm{C}$ fractures who had a posterior malleolus were included in the research. Seventy-seven patients treated from February 2017 to February 2020 were selected for inclusion in the study. Thirty-four were men (44.15\%), and 43 were women (55.85\%). Patients' mean age was 40.5 years, ranging from 23 to 75 years.

Patients underwent frontal and profile radiographs of the ankle ${ }^{(5)}$ and then computed tomography $(\mathrm{CT})^{(6)}$ with axial, sagittal, and coronal planes and 3D reconstruction ${ }^{(7,8)}$. Patients presenting with ankle dislocation or sub-dislocation were underwent reduction by the on-call team at the time of consultation, and osteosyntesis was scheduled for a later date.

This study was approved by the institution's ethical committee.

Inclusion criteria:

- 44B ankle fractures with posterior malleolus

- 44C ankle fractures with posterior malleolus

- Age above 18 years old

Exclusion criteria:

- Exposed fractures

- Previous surgeries on the same ankle

- Age below 18 years old

Data were collected through a review of clinical histories and analysis of pre-and post-operative radiographies, along with $C T^{(9)}$. Data were interpreted as a lesion pattern contemplating items considered essential in these injuries: the size of the posterior fragment, syndesmotic stability, and joint congruence.

The 2 planes used to classify fractures were:

Sagittal. On radiological images, the site of the greatest joint involvement is identified. A straight line is drawn from the most anterior point on the cartilage to its most posterior point and divided into 4 parts (Figure 1). If the fracture compromises at least $25 \%$ of the joint at this level, a posterior approach will be required.

Axial: A section running through $0.5 \mathrm{~cm}$ proximally to the tibial joint cartilage is randomly selected, a measure similar to that presented by Bartoníček et al., in $2019(0.4 \mathrm{~cm})^{(10)}$. A straight line is drawn from the most anterior point on the notch to its most posterior point and divided into 4 parts (Figure 1). If the fracture compromises at least $25 \%$ of this line, posterior open reduction and internal fixation of this fragment are required.

The size of the fracture fragment is a determining factor to perform the classification. Cadaveric studies confirm that fractures lower than $25 \%$ do not compromise joint stability per se ${ }^{(11)}$ and reduce the tibioastragalar contact area by only $4 \%$. Fragments greater than $25 \%$ do generate a major change in the distribution of ankle loads and thus may lead to arthrosis in the medium or long term ${ }^{(12,13)}$. The 2015 series by Drijfhout van
Hooff et al. with 131 patients is the most extensive published and is consistent with this statement ${ }^{(14)}$.

Intra-operative findings were compared with these patterns, and based on the study, the 77 operated patients were regrouped in a novel classification as defined by the therapeutic algorithm.

In terms of surgical approach, the most frequently used was the classical posterolateral one ${ }^{(15-17)}$. The incision was equidistant to 2 lines running through the lateral edge of the Achilles heel and the posterior edge of the fibula. The muscle belly of the flexor hallux longus is medially retracted, allowing for the directing visualization of the fracture fragment in the posterior malleolus. It is important to consider the route of the external saphenous nerve.

Another approach used was the modified posteromedial one. It das performed only in patients whose posterolateral malleolar fracture was associated with a posteromedial mass. This approach is performed between the posterior edge of the medial malleolus and the internal edge of the Achilles heel. It is crucial to identify the flexor hallux longus and always take it as a reference so as to protect the posterior tibial neurovascular bundle.

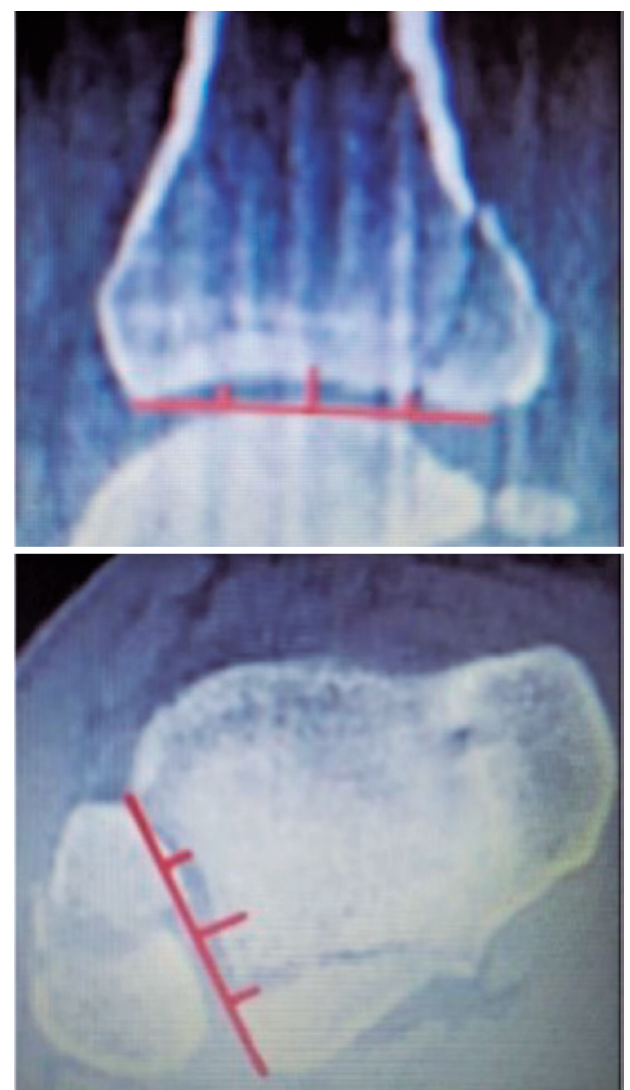

Figure 1. Coronal plane (above) and axial plane (below) to classify each fracture as larger or smaller than $25 \%$. 


\section{Results}

We analyzed the CT scans of all study patients and anatomically recognized two fracture groups, based on the sagittal plane of the ankle and/or axial plane of the syndesmosis, according to the tibial involvement on the joint surface:

1. Fractures affecting less than $25 \%$

2. Fractures affecting more than $25 \%$

Based on the obtained results, we propose a classification with a treatment algorithm.

- Type 1. Fragments compromising less than $25 \%$ of the joint surface on the CT sagittal plane and/or axial plane of the syndesmosis. This fracture pattern always requires investigating syndesmotic stability. We conducted the stress maneuver with external rotation and dorsal ankle flexion. The result may be:

A. Stable fractures: do not require reduction or syndesmotic stabilization (Figure 2). We identified 6 patients (7.79\%) in this group.

B. Unstable fractures: If instability is diagnosed, it should be treated using suprasyndesmotic screws(18) or syndesmotic button, according to the surgeon's preference (Figure 3 ). 26 patients (33.76\%) of our sample belonged to this group.

There is no indication of a posterior approach or direct reduction for posterior malleolar fractures affecting less than $25 \%$ of the joint.
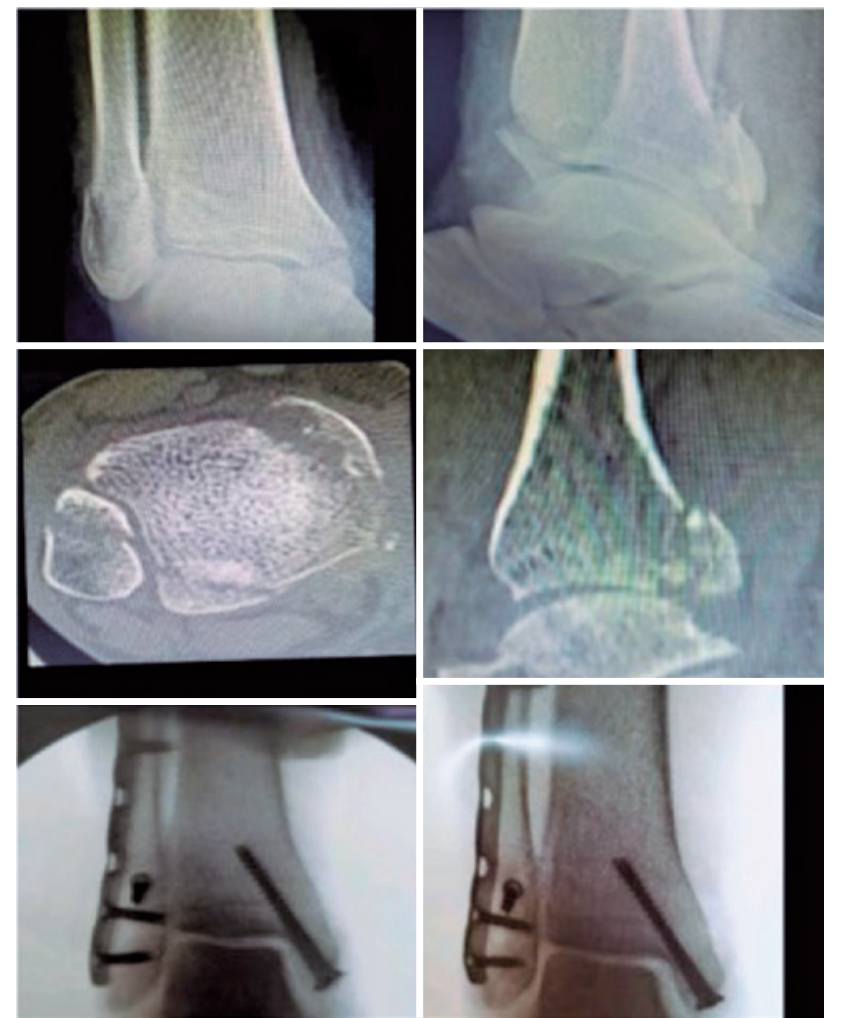

Figure 2. Type 1A fracture with untreated syndesmosis. Below is the intraoperative control of syndesmotic opening.
- Type 2. The fracture fragment compromises the joint in at least $25 \%$ on the sagittal plane of the ankle or axial plane of the syndesmosis.

Within this group, we recognize 3 fracture patterns: a) Non-displaced fractures; b) Displaced fractures; and c) Comminuted fractures:

A. These fractures consist of large non-displaced fragments on TC scans. Fixation with 2 percutaneous screws from posterolateral to anteromedial with the patient in the lateral position (Figure 4), similarly to the way we treat non-displaced talar neck fractures. The stability provided by the 2 screws in the fragment is significantly similar to that provided by an anti glide plate ${ }^{(19,20)}$, which means that it is unnecessary to perform a fixation approach.

There were 4 patients (5.19\%).

B. Displaced fractures always require a posterior approach and stabilization with a postero-external anti glide plate(21) (Figure 5). This group comprised 46 patients (59.74\%), thus being the more frequent pattern of posterior malleolar fractures.

C. Comminuted fractures compromising more than $25 \%$ of the joint should always be fixed by a posterolateral approach and placement of anti-glide plate. This group included 5 patients (6.49\%). The difference from group $2 \mathrm{~B}$ is that these fractures did not present with intermediate fragments, which should often be approached since they are found to be interposed and may hamper the reduction of the main fragment.

It is important to assess 3 topics that may be presented in displaced fractures:
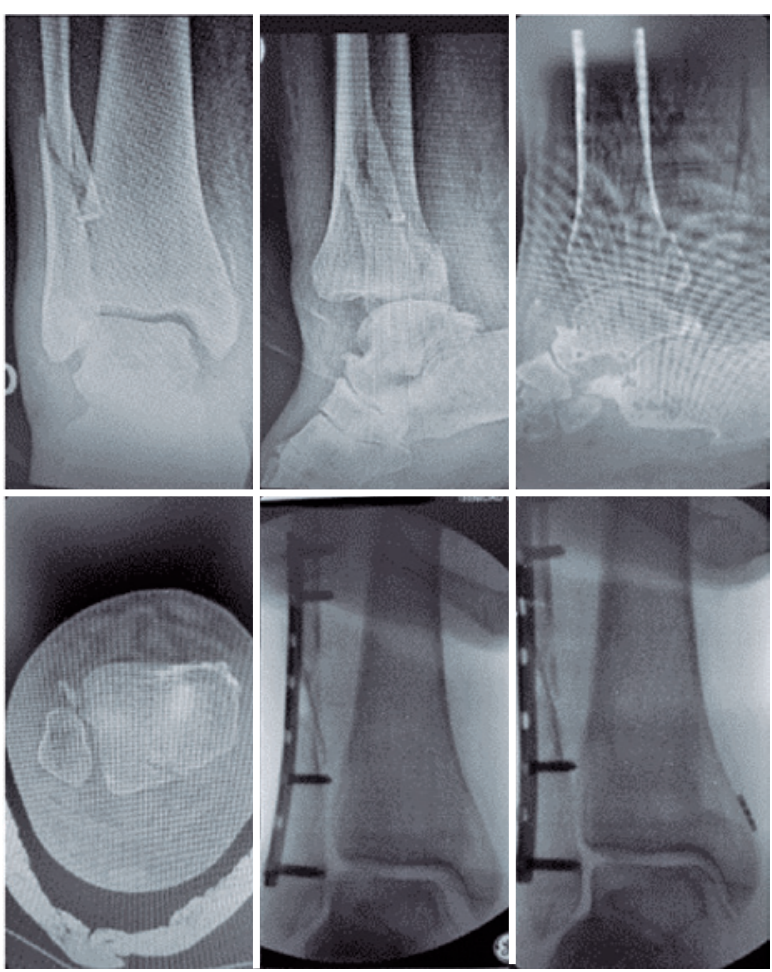

Figure 3. Type 1B fracture stabilized with a syndesmotic button. 

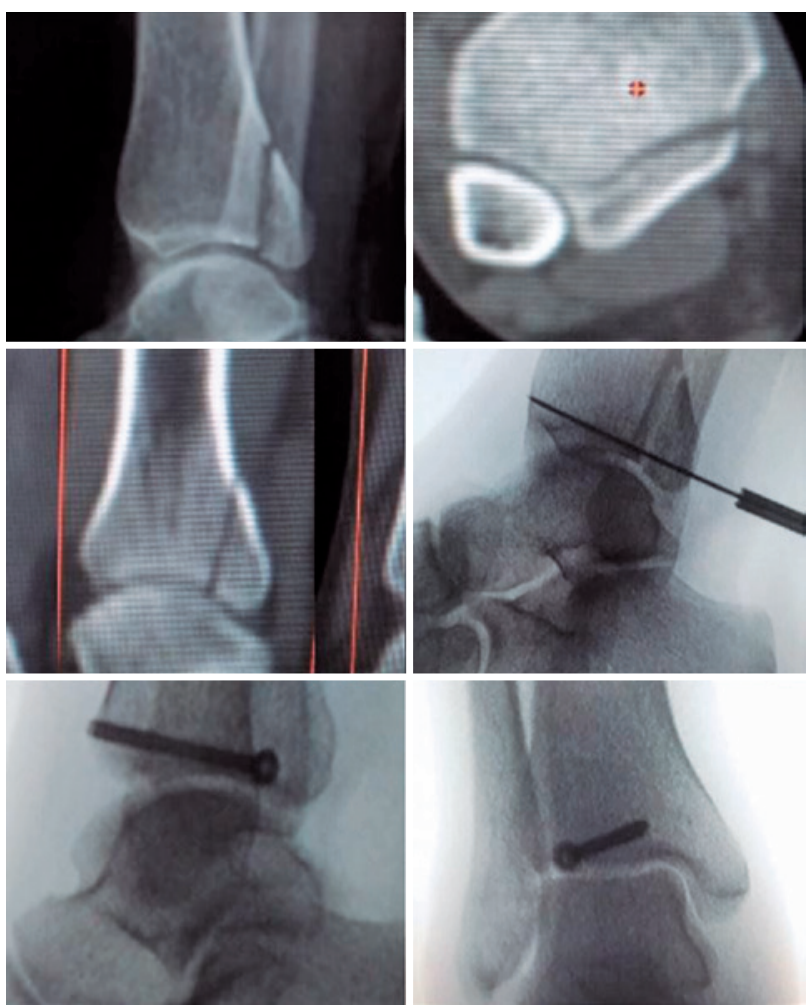

Figure 4. Type 2A fracture treated percutaneously.
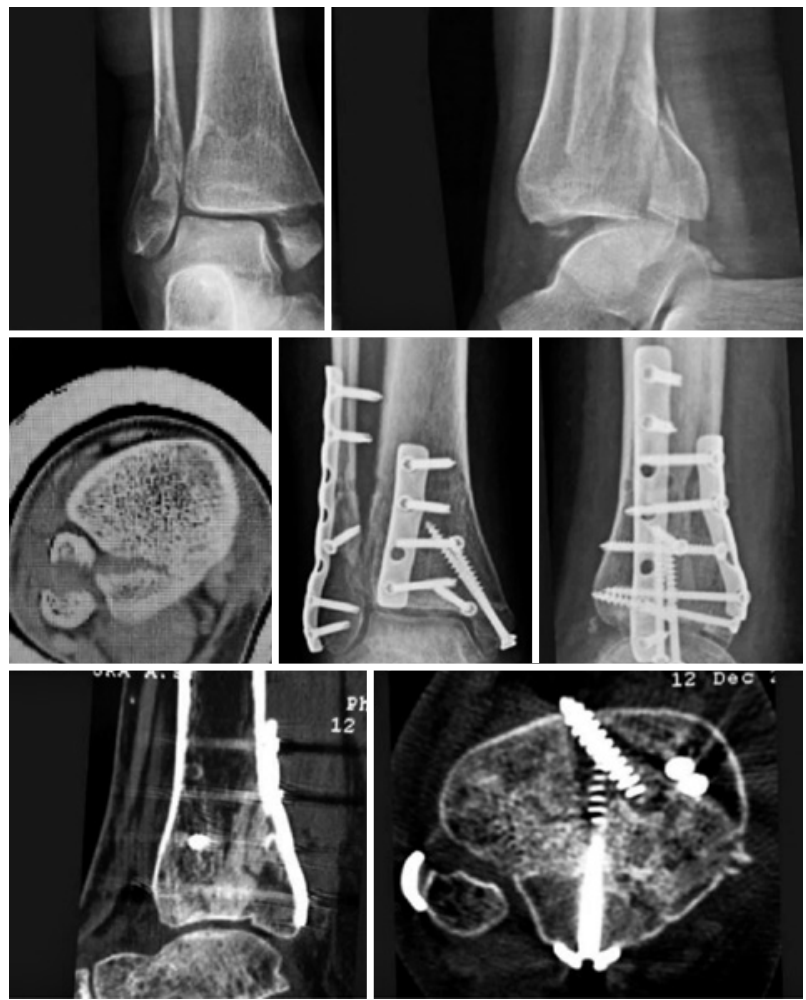

Figure 5. Type 2B fracture treated with an antiglide plate.
1. The existence of an interposed fragment;

\section{Syndesmotic instability;}

3. Incongruence on CT scan.

None of these 3 subtypes leads to changes in the therapeutic focus, but they should be considered when scheduling osteosynthesis and evaluating intra and post-operative radiographic controls.

\section{Discussion}

Posterior malleolar fractures have gained importance and prominence with regard to definite outcomes of ankle fractures. Their contribution to the reduction and stability of fractures is directly related to their long-term prognosis.

It is crucial to classify posterior malleolar fractures into posteromedial and posterolateral, according to their anatomical position, since the first has different behavior and a different approach. We established the lateral edge of the posterior tibial tendon groove as the boundary between the two types of fractures.

They follow different fracture patterns.

The posteromedial fragment usually extends up to the medial malleolus (posterior colliculus) and is closely related to the posterior tibial tendon and its sheath ${ }^{(22)}$. One of these characteristics that differs these fractures from that of the posterolateral malleolus is the fact that their reduction is impossible to be performed through ligamentotaxis ${ }^{(23)}$. We believe that posteromedial fractures should be classified as a subtype of medial malleolar fractures and not as part of posterior malleolar fractures. When these fractures are associated with syndesmotic instability, they display a lesion association with anterior and interosseous syndesmotic ligaments. In the context of a complex ankle fracture, this type of associated syndesmotic instability would require fixation with suprasyndesmotic screws or suprasyndesmotic suture button, according to surgeon practice and experience ${ }^{(24)}$.

Conversely, posterolateral malleolar fractures exhibit a different pattern. They consist of those from the lateral edge of the malleolar groove to the external malleolus. These fractures may be reduced through ligamentotaxis. The posterior-inferior and transverse ligaments of the tibiofibular syndesmosis are inserted at the level of the lateral edge of the posterolateral malleolus; therefore, an adequate reduction and stable fixation of fractures affecting this site usually leads to syndesmotic stability.

The most relevant classification was those proposed by Bartonicek et al. in 2014, consisting of 5 types of fragments ${ }^{(25,26)}$ : type 1, extraincisural fragment; type 2, posterolateral fragment; type 3, two-part fragment, posteromedial and posterolateral; type 4, large posterolateral triangular fragment; and type 5, osteoporotic fragments. Mason et al. presented another classification in $2017^{(27)}$, which dividing fractures into 3 types: type 1, extra-articular; type 2, posterolateral triangular fragment; and type 3, a fragment characterized by a coronal plane fracture line involving the whole posterior plafond.

These classifications are more descriptive and anatomical, but do not present a treatment protocol; hence, we believe it is important not only to describe each fracture but also to standardize treatment for each of their types. 
The present study is based on the interpretation of radiographic and tomographic images of posterior malleolar fractures to develop an anatomical classification and report a related therapeutic algorithm and thus achieve anatomical reduction and syndesmotic stability (Figure 6).

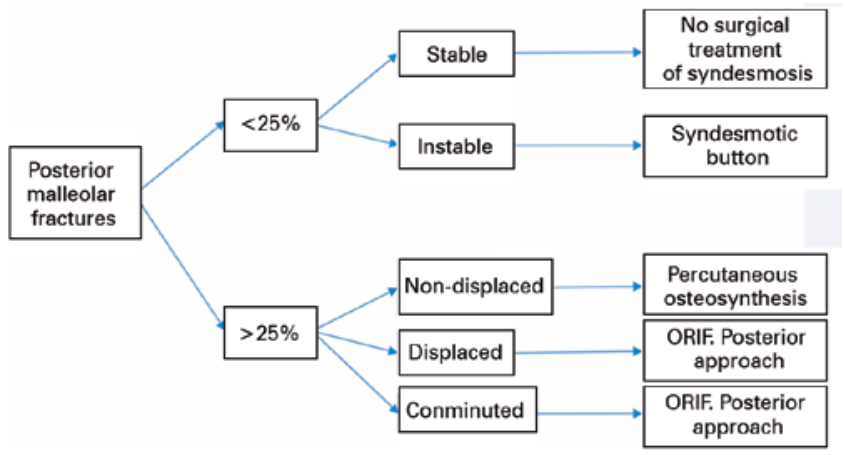

Figure 6. Classification of posterior malleolar fractures
The limitation of this study relies on its retrospective design. Although the sample is important, the study is based on the authors' interpretation of radiographic and tomographic images for the identification of lesion patterns.

\section{Conclusion}

This study proposes a classification and a treatment algorithm for posterior malleolar fractures that we believe will be helpful for its interpretation and decision-making on the need to perform a posterior approach, prioritizing the anatomical reduction of the joint fragment and resolution of syndesmotic instability linked to each fracture pattern using the most simple and effective method.

Each fracture pattern is associated with a surgical approach that we consider necessary, with the most convenient type of osteosynthesis for each case, and with the resolution of syndesmotic instability simply and algorithmically.

Authors' contributions: Each author contributed individually and significantly to the development of this article: DY *(https://orcid.org/OOOO-00029542-6914) = Conceived and planned the activity that led to the study, and approved the final version; IM *(https://orcid.org/0000-0002-9452-0175) = bibliographic review, interpreted result of study, and approved the final version; FA *(https://orcid.org/0000-0001-6577-8911) = participated in the review processand and clinical examination, and approved the final version; SS *(https://orcid.org/0000-0003-0432-8102) = data collection, biographic review, and approved the final version; JD*(https://orcid.org/0000-0002-5733-6766) = performed the surgeries; data collection, formatting of the article, and approved the final version. ${ }^{*}$ ORCID (Open Researcher and Contributor ID) iD).

\section{References}

1. Jaskulka RA, Ittner G, Schedl R. Fractures of the posterior tibial margin: their role in the prognosis of malleolar fractures. J Trauma. 1989;29(11):1565-70.

2. Bartonícek J. Avulsed posterior edge of the tibia. Earle's or Volkmann's triangle? J Bone Joint Surg Br. 2004;86(5):746-50.

3. Fuchs CC, Scharplatz D. [Should Volkmann's great triangle be screwed?]. Helv Chir Acta. 1989;56(1-2):259-61.

4. van den Bekerom MP, Haverkamp D, Kloen P. Biomechanical and clinical evaluation of posterior malleolar fractures. A systematic review of the literature. J Trauma. 2009;66(1):279-84.

5. Neumaier Probst E, Maas R, Meenen NM. Isolated fracture of the posterolateral tibial lip (Volkmann's triangle). Acta Radiol. 1997; 38(3):359-62.

6. Ferries JS, DeCoster TA, Firoozbakhsh KK, Garcia JF, Miller RA. Plain radiographic interpretation in trimalleolar ankle fractures poorly assess posterior fragment size. J Orthop Trauma. 1994; 8(4):328-31.

7. Hartford JM, Gorczyca JT, McNamara JL, Mayor MB. Tibiotalar contact area. Contribution of posterior malleolus and deltoid ligament. Clin Orthop Relat Res. 1995;(320):182-7.

8. Büchler L, Tannast M, Bonel HM, Weber M. Reliability of radiologic assessment of the fracture anatomy at the posterior tibial plafond in malleolar fractures. J Orthop Trauma. 2009;23(3):208-12.
9. Haraguchi $\mathrm{N}$, Haruyama $\mathrm{H}$, Toga $\mathrm{H}$, Kato $\mathrm{F}$. Pathoanatomy of posterior malleolar fractures of the ankle. J Bone Joint Surg Am. 2006;88(5):1085-92.

10. Bartoníček J, Rammelt S, Kašper Š, Malík J, Tuček M. Pathoanatomy of Maisonneuve fracture based on radiologic and CT examination. Arch Orthop Trauma Surg. 2019;139(4):497-506.

11. Harper MC. Talar shift. The stabilizing role of the medial, lateral, and posterior ankle structures. Clin Orthop Relat Res. 1990;(257):177-83.

12. Shi HF, Xiong J, Chen YX, Wang JF, Qiu XS, Huang J, et al. Comparison of the direct and indirect reduction techniques during the surgical management of posterior malleolar fractures. BMC Musculoskelet Disord. 2017;18(1):109.

13. Heim UF. Trimalleolar fractures: late results after fixation of the posterior fragment. Orthopedics. 1989;12(8):1053-9.

14. Drijfhout van Hooff CC, Verhage SM, Hoogendoorn JM. Influence of fragment size and postoperative joint congruency on long-term outcome of posterior malleolar fractures. Foot Ankle Int. 2015; 36(6):673-8

15. Abdelgawad AA, Kadous A, Kanlic E. Posterolateral approach for treatment of posterior malleolus fracture of the ankle. J Foot Ankle Surg. 2011;50(5):607-11.

16. Choi JY, Kim JH, Ko HT, Suh JS. Single oblique posterolateral approach for open reduction and internal fixation of posterior 
malleolar fractures with an associated lateral malleolar fracture. J Foot Ankle Surg. 2015;54(4):559-64.

17. Tornetta P 3rd, Ricci W, Nork S, Collinge C, Steen B. The posterolateral approach to the tibia for displaced posterior malleolar injuries. J Orthop Trauma. 2011;25(2):123-6.

18. Miller AN, Carroll EA, Parker RJ, Helfet DL, Lorich DG. Posterior malleolar stabilization of syndesmotic injuries is equivalent to screw fixation. Clin Orthop Relat Res. 2010;468(4):1129-35.

19. Wang X, Yin J, Zhang C, Wang C, Geng X, Ma X, et al. Biomecânico study of screw fixation and plate fixation of a posterior malleolar fracture in a simulation of the normal gait cycle. Foot Ankle Int. 2017;38(10):1132-8.

20. Erdem MN, Erken HY, Burc H, Saka G, Korkmaz MF, Aydogan M. Comparison of lag screw versus buttress plate fixation of posterior malleolar fractures. Foot Ankle Int. 2014;35(10):1022-30.

21. Gardner MJ, Brodsky A, Briggs SM, Nielson JH, Lorich DG. Fixation of posterior malleolar fractures provides greater syndesmotic stability. Clin Orthop Relat Res. 2006;447:165-71.
22. Weber M. Trimalleolar fractures with impaction of the posteromedial tibial plafond: implications for talar stability. Foot Ankle Int. 2004 25(10):716-27.

23. Ebraheim NA, Taser F, Shafiq Q, Yeasting RA. Anatomical evaluation and clinical importance of the tibiofibular syndesmosis ligaments. Surg Radiol Anat. 2006;28(2):142-9.

24. Ebraheim NA, Taser F, Shafiq Q, Yeasting RA. Anatomical evaluation and clinical importance of the tibiofibular syndesmosis ligaments. Surg Radiol Anat. 2006;28(2):142-9.

25. Bartoníček J, Rammelt S, Kostlivý K, Vaněček V, Klika D, Trešl I. Anatomy and classification of the posterior tibial fragment in ankle fractures. Arch Orthop Trauma Surg. 2015;135(4):505-16.

26. Bartoníček J, Rammelt S, Tuček M, Naňka O. Posterior malleolar fractures of the ankle. Eur J Trauma Emerg Surg. 2015;41(6):587-600.

27. Mason LW, Marlow WJ, Widnall J, Molloy AP. Pathoanatomy and associated injuries of posterior malleolus fracture of the ankle. Foot Ankle Int. 2017;38(11):1229-35. 